ISSN 1112-9867

http://www.jfas.info

\title{
TEMPERATURE-DEPENDENT VISCOSITY EFFECTS ON FREE CONVECTION \\ FLOW OVER A VERTICAL MOVING CYLINDER WITH CONSTANT AXIAL VELOCITY UNDER CONSIDERATION OF RADIAL MAGNETOHYDRODYNAMIC
}

\author{
F. Rouzbahani ${ }^{1, *}$ and R. Alizadeh ${ }^{2}$ \\ ${ }^{1}$ Department of Mechanical Engineering, Hamedan Branch, Islamic Azad University, \\ Hamedan, Iran \\ ${ }^{2}$ Department of Mechanical Engineering, Quchan Branch, Islamic Azad University, Quchan, \\ Iran
}

Published online: 05 June 2016

\begin{abstract}
In this present work the effects of temperature-dependent viscosity on MHD free convection flow over a vertical moving cylinder with, the surface of which is exposed to constant wall temperatures. Numerical solutions of the Navier-Stokes equations and energy equation are derived in this problem. A reduction of these equations is obtained by use of appropriate transformations. The semi-similar solution of the Navier-Stokes equations and energy equation has been obtained numerically using by the implicit finite difference scheme of Crank-Nicolson's type. The velocity and temperature profiles for constant values of cylinder axial velocity are plotted. The influence of the Grashof number, Prandtl number, viscosity-variation parameter, thermal conductivity-variation parameter and magnetic parameter on free convection flow and heat transfer is discussed.
\end{abstract}

Keywords: MHD free convection; moving cylinder; temperature-dependent.

Author Correspondence, e-mail: f.rouzbahani@srbiau.ac.ir

doi: http://dx.doi.org/10.4314/jfas.8vi2s.56 


\section{INTRODUCTION}

A study of the flow of electrically conducting fluid in presence of magnetic field is important from the technical point of view and such types of problems have received much attention by many researchers. Natural convection flow of a dissipative fluid over a vertical moving cylinder with time-dependent axial velocity, thermal conductivity and viscosity depending on temperature under the influence of a uniform transverse magnetic field is an important problem relevant to many engineering applications. In the glass and polymer industries, hot filaments, which are considered as vertical cylinders, are cooled as they pass through the surrounding environment. Free convection flows are of great interest in a number of industrial applications such as fiber and granular insulation, geothermal systems etc. Buoyancy is also of importance in an environment where differences between land and air temperatures can give rise to complicated flow patterns. Magnetohydrodynamic has attracted the attention of a large number of scholars due to its diverse applications. In astrophysics and geophysics, it is applied to study the stellar and solar structures, interstellar matter, radio propagation through the ionosphere etc. In engineering it finds its application in MHD pumps, MHD bearings etc. Convection in porous media has applications in geothermal energy recovery, oil extraction, thermal energy storage and flow through filtering devices. The phenomena of mass transfer are also very common in theory of stellar structure and observable effects are detectable, at least on the solar surface. The study of effects of magnetic field on free convection flow is important in liquid-metals, electrolytes and ionized gases. The thermal physics of hydromagnetic problems with mass transfer is of interest in power engineering and metallurgy.

Sparrow and Gregg [1] first studied the heat transfer from vertical cylinder. Goldstein and Briggs [2] presented an analysis of the transient free convective flow past vertical flat plate and circular cylinder to a surrounding initially quiescent fluid by employing Laplace transform technique. Nagendra et al. [3] presented a boundary layer analysis of free convection heat transfer from a vertical cylinder with uniform heat flux at its surface. An experimental and analytical study is reported by Evas et al. [4] for transient natural convection in a vertical cylinder. Velusamy and Grag [5], given a numerical solution for the transient 
natural convection over a heat generating vertical cylinder. The study of flow problems, which involve the interaction of several phenomena, has a wide range of applications in the field of science and technology. One such study is related to the effects of MHD free convection flow, which plays an important role in geophysics, astrophysics and petroleum industries. Michiyoshi et al. [6] considered natural convection heat transfer from a horizontal cylinder to mercury under a magnetic field. Magnetic field effect on a moving vertical cylinder with constant heat flux was given by Ganesan and Loganathan [7]. Free convection flow involving coupled heat and mass transfer occurs frequently in nature. It occures not only due to temperature differences, but also due to concentration differences or a combination of these two, for example, in atmospheric flows there exist differences in the $\mathrm{H}_{2} \mathrm{O}$ concentration. A few representative fields of interest in which combined heat and mass transfer plays an important role are designing of chemical processing equipment, formation and dispersion of fog, distribution of temperature and moisture over agricultural fields and groves of fruit trees, crop damage due to freezing, and environmental pollution.The effects of heat and mass transfer on natural convection flow over a vertical cylinder was studied by Chen and Yuh [8]. Combined heat and mass transfer effects on moving vertical cylinder for steady and unsteady flows were analyzed by Takhar et al. [9] and Ganesan and Loganathan [10] respectively. Gebhart \& Pera [11] analysed the steady combined buoyancy effects of thermal and mass diffusion on vertical natural convection flows. Bottemanne [12] studied the combined effect of heat and mass transfer in the steady laminar boundary layer of a vertical cylinder placed in still air. Elgazery and Hassan [13] presented a numerical study of radiation effect on MHD transient mixed convection flow over a moving vertical cylinder with constant heat flux through a porous medium. Reddy and Reddy $[14,15]$ presented a numerical study of the interaction of radiation and mass transfer effects on unsteady MHD free convection flow past a semi-infinite moving vertical cylinder by employing finite-difference scheme of Crank-Nicolson type. Recently R. K. Deka and A.Paul [16] studied the unsteady free convection flow past a moving vertical cylinder with constant temperature by employing Laplace transform technique. A numerical solution for the transient natural convection flow over a vertical cylinder under the combined buoyancy effect of heat and mass transfer was given by Ganesan and Rani [17], by employing 
an implicit fnite-difference scheme. Shanker and Kishan [18] presented the effect of mass transfer on the MHD flow past an impulsively started infinite vertical plate. Ganesan and Rani [19] studied the MHD unsteady free convection flow past a vertical cylinder with heat and mass transfer. In the context of space technology and in processes involving high temperatures, the effects of radiation are of vital importance. On assuming that the viscosity of the fluid is linear functions of temperature, a semi-empirical formula was proposed by Charraudeau [20] which is appropriate for small Prandtl number. Studies of free convection flow along a vertical cylinder or horizontal cylinder are important in the field of geothermal power generation and drilling operations where the free stream and buoyancy induced fluid velocities are of roughly the same order of magnitude. Many researchers such as Arpaci [21], Cess [22], Cheng and Ozisik [23], Raptis [24], Hossain and Takhar [25, 26] have investigated the interaction of thermal radiation and free convection for different geometries, by considering the flow to be steady. The unsteady flow past a moving vertical plate in the presence of free convection and radiation were studied by Das et al. [27]. Radiation and mass transfer effects on two-dimensional flow past an impulsively started isothermal vertical plate were studied by Ramachandra Prasad et al. [28]. The combined radiation and free convection flow over a vertical cylinder was studied by Yih [29]. Radiation and mass transfer effects on flow of an incompressible viscous fluid past a moving vertical cylinder was studied by Ganesan and Loganathan [30]. MHD natural convection flow from an isothermal horizontal circular cylinder under consideration of temperature dependent viscosity were studied by Molla et al.[31]. Rahman et al [32] have investigated the effects of temperature dependent thermal conductivity on MHD free convection flow along a vertical flat plate with heat conduction. MHD free convection flow along a vertical plate with thermal conductivity and viscosity depending on temperatur was studied by Rehena and Alim [33].

The object of the present paper is to study the free convection flow of a dissipative fluid on a vertical moving cylinder with time-dependent axial velocity, thermal conductivity and viscosity depending on temperature, under the influence of a uniform transverse magnetic field in the presence of constant wall temperatures. The dimensionless governing equations are solved by using an implicit finite difference scheme of Crank-Nicolson's type. 


\section{MATHEMATICAL ANALYSIS}

Consider the free convection flow of a vertical moving cylinder with viscosity depending on temperature in constant wall temperatures (Fig. 1) under the action of a transverse magnetic field. Under these assumptions and Boussinesq's approximation, the flow is governed by the following system of equations:

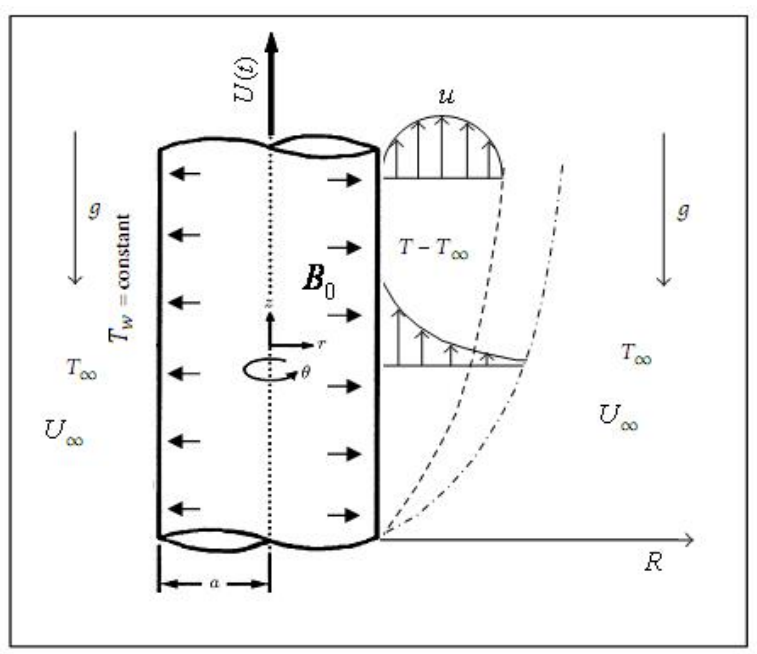

Fig.1. Sketch of the physical model

Continuity equation:

$\frac{\partial(r u)}{\partial z}+\frac{\partial(r v)}{\partial r}=0$

Momentum equation:

$$
\frac{\partial u}{\partial t}+u \frac{\partial u}{\partial z}+v \frac{\partial u}{\partial r}=v\left(\frac{\partial^{2} u}{\partial r^{2}}+\frac{1}{r} \frac{\partial u}{\partial r}\right)+\beta g\left(T-T_{\infty}\right)-\frac{\sigma B_{0}^{2}}{\rho} u
$$

Energy equation:

$$
\frac{\partial T}{\partial t}+u \frac{\partial T}{\partial z}+v \frac{\partial T}{\partial r}=\frac{k}{\rho C_{P}} \frac{1}{r} \frac{\partial}{\partial r}\left(r \frac{\partial T}{\partial r}\right)
$$

here $\mathrm{u}$ and $\mathrm{v}$ are components of the velocity in $\mathrm{z}$ and $\mathrm{r}$ directions, respectively, $\mathrm{t}$ is the time, $v$ is the kinematic viscosity, $\beta$ is the volumetric coefficient of thermal expansion, $g$ is the acceleration due to gravity, $\rho$ is the density, $\sigma$ fluid electrical conductivity, $B_{0}$ is magnetic induction, $\alpha$ is fluid thermal diffusivity, $c_{p}$ is specific heat at constant pressure, 
$T$ is the temperature and $T_{\infty}$ is the temperature of the fluid far away from the cylinder.

The necessary initial and boundary conditions are:

$$
\begin{array}{lll}
t \leq 0 & : u=0, v=0 \quad, T=T_{\infty} \\
t>0 \quad: & u=0, v=0, T=T_{\infty} \quad \text { at } \quad z=0 \\
t>0 \quad: \quad u=u_{0} . U(t), v=0, T=T_{w} \quad \text { at } \quad r=a \\
t>0 \quad: u=0, T \rightarrow T_{\infty} \quad \text { at } \quad r \rightarrow \infty
\end{array}
$$

Out of the many forms of viscosity variation, which are available in the literature, we will consider only following form proposed by Charraudeau [20]

$$
\mu=\mu_{\infty}\left[1+\gamma^{*}\left(T-T_{\infty}\right)\right]
$$

Where $\mu_{\infty}$ is the viscosity of the ambient fluid and $\gamma^{*}$ is defined as follows

$$
\gamma^{*}=\frac{1}{\mu_{f}}\left(\frac{\partial \mu}{\partial T}\right)_{f}
$$

Here $f$ denotes the film temperature of the fluid.

Now introduce the following non dimensional quantities:

$$
\begin{gathered}
\tau=\frac{v_{\infty}}{a^{2}}, \quad U=\frac{u}{u_{0}}, \quad V=\frac{v \cdot a}{v_{\infty}} \quad, Z=\frac{z \cdot v_{\infty}}{u_{0} \cdot a^{2}}, R=\frac{r}{a}, \quad \gamma=\frac{1}{\mu_{f}}\left(\frac{\partial \mu}{\partial T}\right)_{f}\left(T_{w}-T_{\infty}\right), \\
\theta=\frac{T-T_{\infty}}{T_{w}-T_{\infty}}, G r=\frac{g \beta a^{2}\left(T_{w}-T_{\infty}\right)}{v_{\infty} \cdot u_{0}}, \quad M=\frac{\sigma B_{0}^{2} a^{2}}{\mu_{\infty}}, \operatorname{Pr}=\frac{\mu_{\infty} \cdot C_{p}}{k}, \quad v_{\infty}=\frac{\mu_{\infty}}{\rho}
\end{gathered}
$$

Where a is cylinder radius, $Z$ is the dimensionless axial coordinate, $r$ is the dimensionless radial coordinate perpendicular to $Z, U, V$ is the dimensionless velocities, $\tau$ is the dimensionless time, $\theta$ is the dimensionless temperature, $T_{w}$ is the temperature at the surface, $v_{\infty}$ is the reference kinematic viscosity, $\mathrm{Gr}$ is the thermal Grashof number, $\mathrm{M}$ is the magnetic parameter, Pr is the prandtl number, $\gamma$ is the viscosity-variation parameter.

Continuity equation:

$$
\frac{\partial(R U)}{\partial Z}+\frac{\partial(R V)}{\partial R}=0
$$


Momentum equation:

$$
\frac{\partial U}{\partial \tau}+U \frac{\partial U}{\partial Z}+V \frac{\partial U}{\partial R}=(1+\gamma \theta)\left(\frac{\partial^{2} U}{\partial R^{2}}+\frac{1}{R} \frac{\partial U}{\partial R}\right)+\gamma \frac{\partial U}{\partial R} \frac{\partial \theta}{\partial R}+G r \cdot \theta-M \cdot U
$$

Energy equation:

$$
\frac{\partial \theta}{\partial \tau}+U \frac{\partial \theta}{\partial Z}+V \frac{\partial \theta}{\partial R}=\frac{1}{\operatorname{Pr}}\left(\frac{\partial^{2} \theta}{\partial R^{2}}+\frac{1}{R} \frac{\partial \theta}{\partial R}\right)
$$

The dimensionless boundary conditions become:

$$
\begin{aligned}
& \tau \leq 0 \quad: \quad U=0, V=0 \quad, \quad \theta=0 \\
& \tau\rangle 0 \quad: U=0 \quad, V=0 \quad, \quad \theta=0 \quad \text { at } \quad Z=0 \\
& \tau\rangle 0: U=u_{0} . U(\tau), V=0, \theta=1 \quad \text { at } \quad R=1 \\
& \tau\rangle 0 \quad: \quad U=0 \quad, \theta=0 \quad \text { at } \quad R \rightarrow \infty
\end{aligned}
$$

\section{NUMERICAL SOLUTION OF THE PROBLEM}

The governing equations (7-9) are steady, coupled and non-linear with boundary conditions. An implicit finite-difference technique of Crank-Nicolson has been employed to solve the nonlinear coupled equations, as described (Thomas algorithm) in Carnahan et al [35].The finite difference equations corresponding to equations (7-9) are as follows :

$$
\begin{aligned}
& \frac{U_{i, j}^{n+1}-U_{i-1, j}^{n+1}+U_{i, j}^{n}-U_{i-1, j}^{n}}{2 \Delta Z}+\frac{V_{i, j+1}^{n+1}-V_{i, j-1}^{n+1}+V_{i, j+1}^{n}-V_{i, j-1}^{n}}{4 \Delta R}+\frac{V_{i, j}^{n+1}+V_{i, j}^{n}}{R}=0 \\
& \frac{U_{i, j}^{n+1}-U_{i, j}^{n}}{\Delta \tau}+U_{i, j}^{n} \frac{U_{i, j}^{n+1}-U_{i-1, j}^{n+1}+U_{i, j}^{n}-U_{i-1, j}^{n}}{2 \Delta Z}+V_{i, j}^{n} \frac{U_{i, j+1}^{n+1}-U_{i, j-1}^{n+1}+U_{i, j+1}^{n}-U_{i, j-1}^{n}}{4 \Delta R} \\
& =\left(1+\gamma \cdot \theta_{i, j}^{n}\right)\left(\frac{U_{i, j+1}^{n+1}-2 U_{i, j}^{n+1}+U_{i, j-1}^{n+1}+U_{i, j+1}^{n}-2 U_{i, j}^{n}+U_{i, j-1}^{n}}{2 \Delta R^{2}}+\frac{1}{R} \frac{U_{i, j+1}^{n+1}-U_{i, j-1}^{n+1}+U_{i, j+1}^{n}-U_{i, j-1}^{n}}{4 \Delta R}\right) \\
& +\gamma \cdot\left(\frac{\theta_{i, j+1}^{n+1}-\theta_{i, j-1}^{n+1}+\theta_{i, j+1}^{n}-\theta_{i, j-1}^{n}}{4 \Delta R}\right) \cdot\left(\frac{U_{i, j+1}^{n+1}-U_{i, j-1}^{n+1}+U_{i, j+1}^{n}-U_{i, j-1}^{n}}{4 \Delta R}\right)+G r \frac{\theta_{i, j}^{n+1}+\theta_{i, j}^{n}}{2}-M \frac{U_{i, j}^{n+1}+U_{i, j}^{n}}{2} \\
& \frac{\theta_{i, j}^{n+1}-\theta_{i, j}^{n}}{\Delta \tau}+U_{i, j}^{n} \frac{\theta_{i, j}^{n+1}-\theta_{i-1, j}^{n+1}+\theta_{i, j}^{n}-\theta_{i-1, j}^{n}}{2 \Delta Z}+V_{i, j}^{n} \frac{\theta_{i, j+1}^{n+1}-\theta_{i, j-1}^{n+1}+\theta_{i, j+1}^{n}-\theta_{i, j-1}^{n}}{4 \Delta R}
\end{aligned}
$$




$$
=\frac{1}{\operatorname{Pr}}\left(\frac{\theta_{i, j+1}^{n+1}-2 \theta_{i, j}^{n+1}+\theta_{i, j-1}^{n+1}+\theta_{i, j+1}^{n}-2 \theta_{i, j}^{n}+\theta_{i, j-1}^{n}}{2 \Delta R^{2}}+\frac{1}{R} \frac{\theta_{i, j+1}^{n+1}-\theta_{i, j-1}^{n+1}+\theta_{i, j+1}^{n}-\theta_{i, j-1}^{n}}{4 \Delta R}\right)
$$

The region of integration is considered as a rectangle with sides $Z_{\max }(=1)$ and $R_{\max }(=10)$, where corresponding to $R \rightarrow \infty$ which lies far from the momentum and energy boundary layers. An appropriate mesh sizes considered for the calculation are $\Delta Z=0.01$, $\Delta R=0.05$ and $\Delta \tau=0.005$.

\section{RESULTS AND DISCUSSION}

The velocity and temperature profiles have been computed by using implicit finite difference scheme of Crank-Nicolson's type. The numerical calculations are carried out for the effect of the flow parameters such as time-dependent axial velocity $U(\tau)$, Prandtl number (Pr, thermal Grashof number $(\mathrm{Gr})$, viscosity-variation $\operatorname{parameter}(\gamma)$ and magnetic parameter $(\mathrm{M})$ on the velocity and temperature distribution of the flow fields are presented graphically in figure 2-9. The effects of Grashof number (Gr) on the velocity and temperature profiles for $\gamma=0$ and time-dependent axial velocity of cylinder $U(\tau)=\exp (-\tau)$ are shown in Figs. 2-3. It is observed that the velocity and temperature increases with increase in Grashof number.

Figs 4-5 illustrates the dimensionless velocity and temperature profiles for $\gamma=0$ and time-dependent axial velocity of cylinder $U(\tau)=\exp (-\tau)$ and for magnetic parameter $(\mathrm{M})$. It is obvious that, the velocity and temperature decreases with increases in magnetic parameter. The presence of the transverse magnetic field produces a resistive force the fluid flow. This force is called the Lorentz force, which leads to slow down the motion of electrically conducting fluid, which tends to increase the temperature.

Figs. 6-7 depict the velocity and temperature profiles for $\gamma=0$ and time-dependent axial velocity of cylinder $U(\tau)=\exp (-\tau)$ and for different values of Prandtl number (Pr). It is observed that the velocity and temperature decreases with increase in the Prandtl number.

Figs. 8-9 depict the velocity and temperature profiles for time-dependent axial velocity of 
cylinder $U(\tau)=\exp (-\tau)$ and for different values of viscosity-variation parameter $(\gamma)$. It is observed that the velocity and temperature increases with increase in the viscosity-variation $\operatorname{parameter}(\gamma)$.

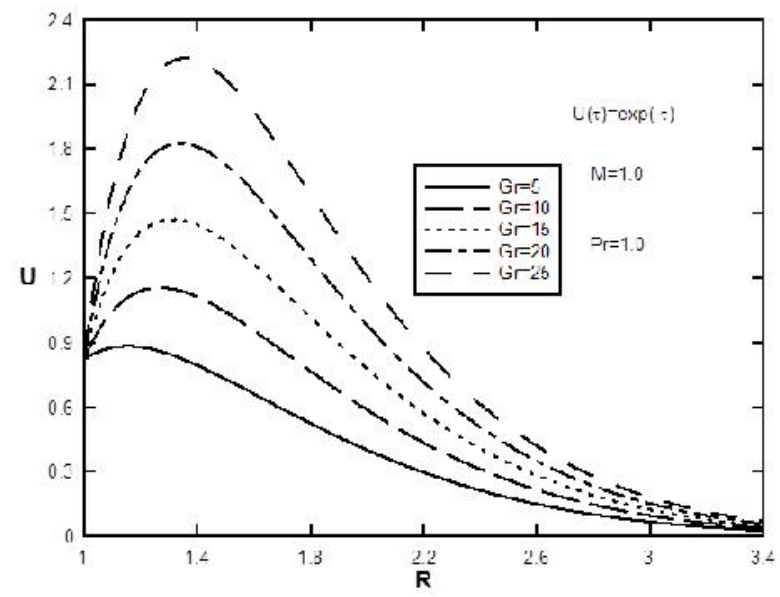

Fig.2. Effect of Grashof number (Gr) on dimensionless velocity

Profiles for $\gamma=0$ and time-dependent axial velocity of cylinder $U(\tau)=\exp (-\tau)$

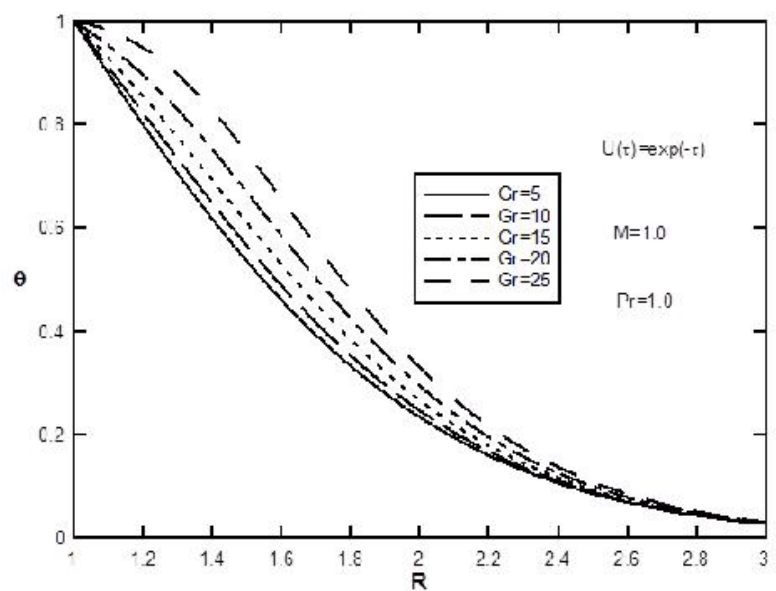

Fig.3. Effect of Grashof number (Gr) on dimensionless temperature Profiles for $\gamma=0$ and time-dependent axial velocity of $\operatorname{cylinder} U(\tau)=\exp (-\tau)$ 


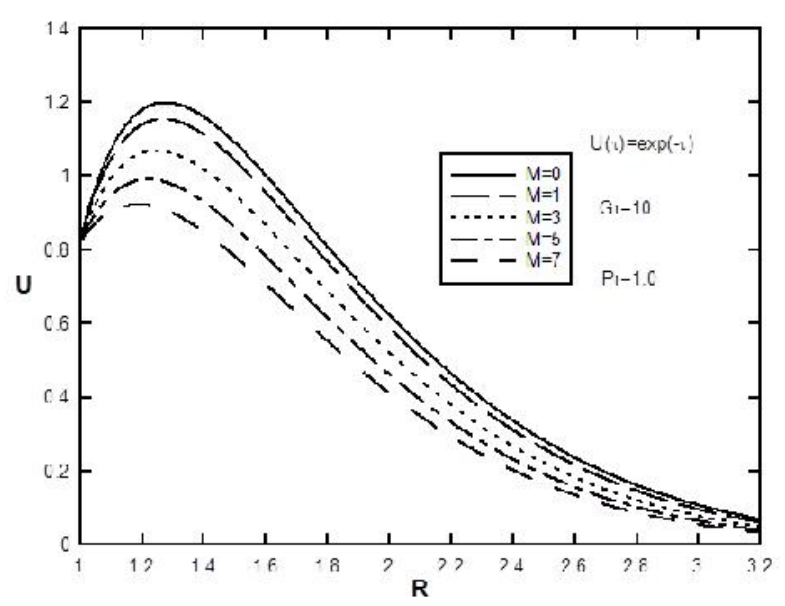

Fig.4. Effect of magnetic parameter (M) on dimensionless velocity

Profiles for $\gamma=0$ and time-dependent axial velocity of cylinder $U(\tau)=\exp (-\tau)$

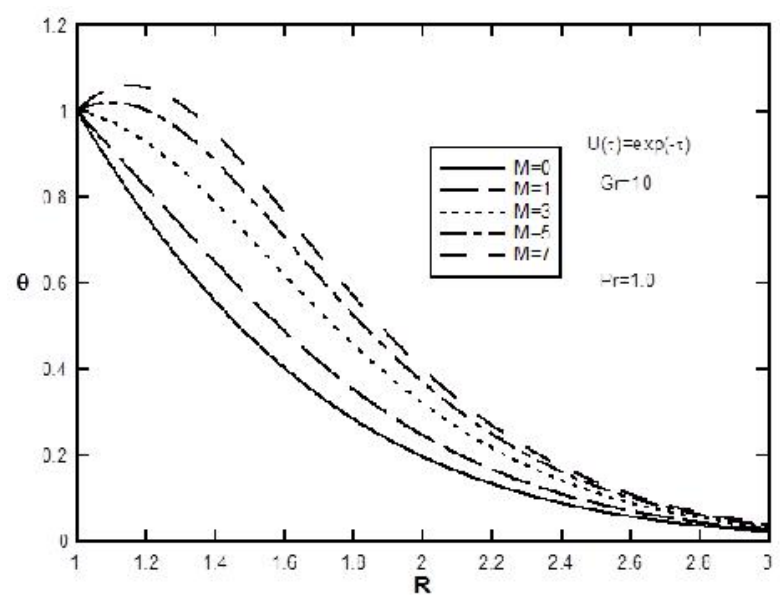

Fig.5. Effect of magnetic parameter $(\mathrm{M})$ on dimensionless temperature Profiles for $\gamma=0$ and time dependent axial velocity of cylinder $U(\tau)=\exp (-\tau)$

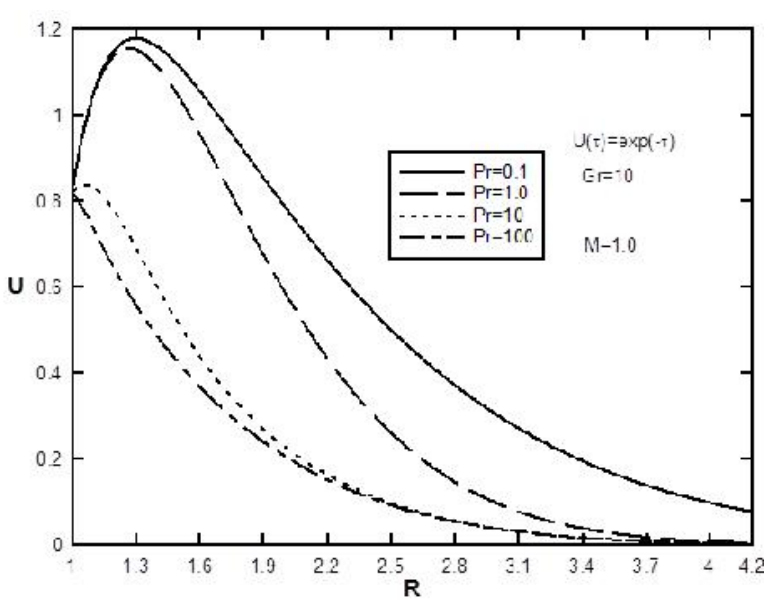

Fig.6. Effect of Prandtl number on dimensionless velocity

Profiles for $\gamma=0$ and time-dependent axial velocity of cylinder $U(\tau)=\exp (-\tau)$ 


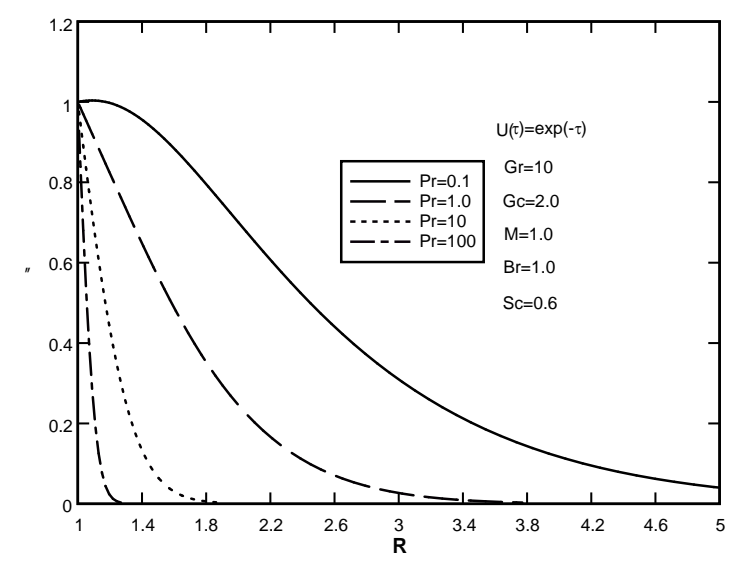

Fig.7. Effect of Prandtl number on dimensionless temperature

Profiles for $\gamma=0$ and time-dependent axial velocity of cylinder $U(\tau)=\exp (-\tau)$

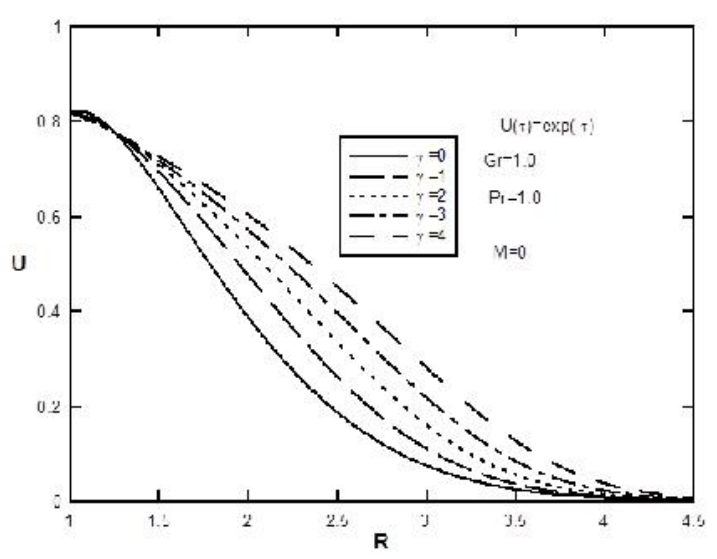

Fig.8. Effect of viscosity-variation parameter $(\gamma)$ on dimensionless velocity

Profiles for time-dependent axial velocity of cylinder $U(\tau)=\exp (-\tau)$

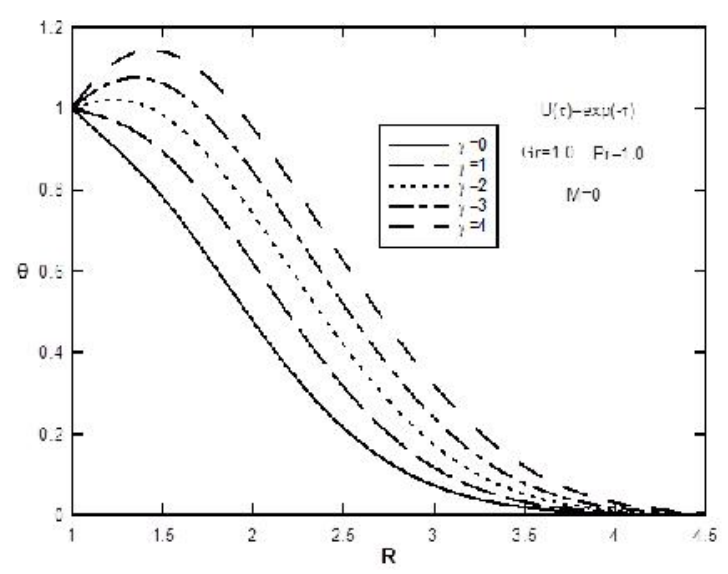

Fig.9. Effect of viscosity-variation parameter $(\gamma)$ on dimensionless temperature

Profiles for time-dependent axial velocity of cylinder $U(\tau)=\exp (-\tau)$ 


\section{CONCLUSIONS}

A numerical study has been carried out to study the MHD free convection flow on a vertical moving cylinder with viscosity depending on temperature, in the presence of constant wall temperatures. The semi-similar solution of the Navier-Stokes equations and energy equation has been obtained numerically using by the implicit finite difference scheme of Crank-Nicolson's type. From the present numerical investigation, following conclusions have been drawn:

1- Velocity increases, temperature decreases with an increase in Grashof number (Gr).

2- Velocity and temperature decreases with an increase in magnetic parameter (M).

3- Velocity and temperature decreases with an increase in Grashof number $(\mathrm{Gr})$.

4- Velocity and temperature increases with an increase in viscosity-variation parameter $(\gamma)$

\section{REFERENCES}

[1] Sparrow, E. M., Gregg, J. L. Laminar free convection heat transfer from the outer surface of a vertical circular cylinder .Trans. ASME, 1956, 78, 1823-1829.

[2] Goldstein, R. J., Briggs, D. G. Transient free convection about a vertical plates and circular cylinders. Trans ASME C: J. Heat Transfer ,1964, 86, 490-500.

[3] Nagendra, H. R., Tirunarayanan, M. A., Ramachandran, A. Laminar Free Convection From Vertical Cylinders $\quad$ With Uniform Heat Flux, J. Heat Transfer, 1970, 92(1), 191-194.

[4] Evan L.B., Reid R.C. and Drake E.M. Transient natural convection in a vertical cylinder, A.I.Ch.E. J, 1968, Vol 14, pp.251-261.

[5] Velusamy K. and Garg V.K. Transient natural convection over a heat generating vertical cylinder, Int. J. Heat Mass Transfer, 1992, Vol.35, pp.1293-1306.

[6] Michiyoshi I., Takahashi I. and Seizawa A. Natural convection heat transfer form a horizontal cylinder to mercury under a magnetic field, Int.J.Heat Mass Transfer, 1976, Vol.19, pp.1021-1029.

[7] Ganesan P. and Loganathan P. Magnetic field effect on a moving vertical cylinder with constant heat flux, Heat Mass Transfer, 2003, Vol.39, pp.381-386. 
[8] Chen T.S. and Yuh C.F. Combined heat and mass transfer in natural convection along a vertical cylinder, Int. J. Heat Mass transfer, 1980, Vol.23, pp.451-461.

[9] Takhar H.S., Chamkha A.J. and Nath G. Combined heat and mass transfer along a vertical cylinder with free stream, Heat Mass Transfer, 2000, Vol.36, pp.237-246.

[10] Ganesan P. and Loganathan P. Unsteady free convection flow over a moving vertical cylinder with heat and mass transfer, Heat Mass Transfer, 2001, Vol.37(1), pp.59-65.

[11] Gebhart, B., Pera, L. The nature of vertical natural convection flows resulting from the combined buoyancy effects of thermal and mass diffusion, Int. J. 1971, Heat Mass Transfer, $14,2025-2050$.

[12] Bottemanne G. A. Experimental results of pure and simultaneous heat and mass transfer by free convection about a vertical cylinder for $\operatorname{Pr}=0: 71$ and $\mathrm{Sc}=0: 63$. Appl Sci Res, 1972, $25,372-382$.

[13] Elgazery, Nasser S., Hassan, M.A. Numerical study of radiation effect on MHD transient mixed convection flow over a moving vertical cylinder with constant heat flux. Communications in Numerical Methods in Engineering, 2008, 24(11), 1183-1202.

[14] Reddy, M. M. G., Reddy, N. B. Radiation and mass transfer effects on unsteady MHD free convection flow of an incompressible viscous fluid past a moving vertical cylinder. Theoretical applied Mechanics, 2009, 36(3), 239-260.

[15] Reddy, M. M. G., Reddy, N. B. Thermal radiation and mass transfer effects on MHD free convection flow past a vertical cylinder with variable surface temperature and concentration. Journal of Naval Architecture and Marine Engineering, 2009, 6(1), 1-24.

[16] R. K. Deka and A. Paul. Unsteady free convection flow past a moving vertical cylinder with constant temperature. IJMA- 2(6), June-2011, page :832-840.

[17] Ganesan P. and Rani H.P. Transient natural convection cylinder with heat and mass transfer, Heat Mass Transfer, 1998, Vol.33, pp.449-455.

[18] Shanker B. and Kishan N. The effects of mass transfer on the MHD flow past an impulsively started infinite vertical plate with variable temperature or constant heat flux, J. Engg..Heat Mass Transfer, 1997, Vol.19, pp.273-278.

[19] Ganesan P. and Rani H.P. Unsteady free convection MHD flow past a vertical cylinder 
with heat and mass transfer, Int. J. Therm. Sci., 2000, Vol.39,pp.265-272.

[20] Charraudeau J. Influence de gradients de properties physiques en convection force application au cas du tube, Int. J. Heat Mass Trans, 1975, 18, 87-95.

[21] Arpaci V.S. Effects of thermal radiation on the laminar free convection from a heated vertical plate, Int. J. Heat Mass Transfer, 1968, Vol.11, pp.871-881.

[22] Cess R.D. Interaction of thermal radiation with free convection heat transfer, Int. J. Heat Mass transfer, 1966, Vol.9, pp.1269-1277.

[23] Cheng E.H and Ozisik M.N. (1972), Radiation with free convection in an absorbing emitting and scattering medium, Int. J. Heat Mass Transfer, Vol.15, pp.1243-1252.

[24] Raptis A. Radiation and free convection flow through a porous medium, Int.Comm. Heat Mass Transfer, 1998, 25(2), pp.289-295.

[25] Hossain M.A. and Takhar H.S. Radiation effects on mixed convection along a vertical plate with uniform surface temperature, Heat Mass Transfer, 1996, Vol.31, pp.243-248.

[26] Hossain M.A. and Takhar H.S. Thermal radiation effects on the natural convection ${ }^{\circ} \mathrm{Ow}$ over an isothermal horizontal plate, Heat Mass Transfer, 1999, Vol.35, pp.321-326.

[27] Das U.N., Deka R. and Soundalgekar V.M. Radiation effects on flow past an impulsively started vertical plate-an exact solutions, J. Theo. Appl. Fluid Mech., 1996, Vol.1(2), pp.111-115.

[28] Ramachandra Prasad V., Bhaskar Reddy N. and Muthucumaraswamy R. Radiation and mass transfer effects on two-dimensional flow past an impulsively started isothermal vertical plate, 2007, Int.J. Thermal Sciences, Vol.46(12), pp.1251-1258.

[29] Yih K.A. Radiation effects on natural convection over a vertical cylinder embedded in porous media, Int. Comm. Heat Mass Transfer, 1999, Vol.26(2), pp.259-267.

[30] Ganesan P. and Loganathan P. Radiation and mass transfer effects on flow of an incompressible viscous fluid past a moving vertical cylinder, Int. J. Heat Mass Transfer, 2002, Vol.45, pp.4281 4288.

[31] Molla, Md. Mamun, Saha, Suvash C., \& Khan, M. A. I. MHD natural convection flow from an isothermal horizontal circular cylinder under consideration of temperature dependent viscosity. Engineering Computations, 2012, (In Press). 
[32] Rahman, M. M., Mamun, A. A., Azim, M. A. and Alim, M. A. Effects of Temperature Dependent Thermal Conductivity on MHD Free Convection Flow along a Vertical Flat Plate with Heat Conduction, Nonlinear Analysis: Modell. and Cont., 2008, Vol. 13, No. 4, pp. $513-524$

[33] Rehena Nasrin and M. A. Alim. MHD free convection flow along a vertical plate with thermal conductivity and viscosity depending on temperatur, Journal of Naval Architecture and Marine Engineering 6, 2009, 72-83.

[34] Brewster M.Q. Thermal radiative transfer and properties, John Wiley \& Sons, New York, 1992.

[35] Carnahan B., Luther H.A. and Wilkes J.O, Applied Numerical Methods, John Wiley \& Sons, New York, 1969.

\section{How to cite this article:}

Rouzbahani F and Alizadeh R. Temperature-dependent viscosity effects on free convection flow over a vertical moving cylinder with constant axial velocity under consideration of radial magnetohydrodynamic. J. Fundam. Appl. Sci., 2016, 8(2S), 407-421. 